\title{
Metformin-induced mixed hepatocellular and cholestatic hepatic injury: case report and literature review
}

This article was published in the following Dove Press journal:

International Journal of General Medicine

17 August 2013

Number of times this article has been viewed

\author{
Tarek Saadi ${ }^{1-3}$ \\ Matti Waterman ${ }^{1-4}$ \\ Heba Yassin ${ }^{3}$ \\ Yaacov Baruch ${ }^{1,4}$ \\ 'Liver Unit, ${ }^{2}$ Department of \\ Gastroenterology, ${ }^{3}$ Department of \\ Internal Medicine A, Rambam Health \\ Care Campus, Haifa, Israel; ${ }^{4}$ The \\ Bruce Rappaport Faculty of Medicine, \\ Technion-Israel Institute \\ of Technology, Haifa, Israel
}

Introduction: Metformin is a first-line drug choice for the treatment of type 2 diabetes mellitus (DM-2). Metformin-induced hepatotoxicity has rarely been reported. We report on a case of metformin-induced mixed hepatocellular and cholestatic liver injury in an elderly patient with DM-2 as well as review and summarize case reports of metformin hepatotoxicity available in English on the PubMed database.

Case: After receiving metformin $850 \mathrm{mg}$ /day for 2 weeks, a 78-year-old male presented with a 10-day history of abdominal pain, vomiting, diarrhea, and jaundice. Laboratory analysis showed severe hepatocellular and cholestatic hepatic injury. Other causes for acute liver injury were ruled out. Discontinuation of metformin treatment led to significant subjective improvement after 1 week, and all hepatic abnormalities resolved by 2 months.

Conclusion: Metformin is an important drug for the treatment of DM-2, which is also used for treatment of patients with fatty liver. It can, however, induce hepatocellular and cholestatic hepatic injury; both physicians and patients should be aware of this potential side effect.

Keywords: metformin, hepatocellular liver injury, cholestasis, hepatotoxicity

\section{Introduction}

Metformin is the current biguanide of choice for the treatment of type 2 diabetes mellitus (DM-2). ${ }^{1}$ Studies have shown that metformin ameliorates hyperglycemia without stimulating insulin secretion, weight gain, or hypoglycemia. ${ }^{2}$ A largescale study found that metformin reduced all-cause mortality in obese patients with DM-2. ${ }^{3}$

Metformin has a multifactorial mechanism of action, but acts primarily by improving insulin sensitivity, with a concomitant decrease in hepatic glucose production and an increase in glucose transport across the skeletal muscle membrane. ${ }^{4,5}$ Despite its clinical efficacy, metformin has been associated with several adverse effects. These are primarily gastrointestinal in nature (diarrhea, nausea, vomiting, bloating, and flatulence), and with long-term use, reductions in serum folic acid and vitamin B12 levels have been reported. ${ }^{4}$ Because of its interference with mitochondrial oxidative processes, its most significant, although rare, side effect is lactic acidosis in the context of significantly chronically decreased renal function. ${ }^{6}$ Other oral antidiabetic agents, such as acarbose, ${ }^{7}$ gliclazide ${ }^{8}$ and some of the thiazolidinediones (mainly troglitazone), have been implicated in liver toxicity. ${ }^{9}$ In the case of metformin, hepatotoxicity has been only rarely reported. ${ }^{10,11}$

We report a case of acute hepatocellular and cholestatic jaundice due to metformin therapy and review the literature.
Correspondence: Tarek Saadi

Liver Unit, Rambam Health Care

Campus, POB 9602, Haifa, 31 096, Israel

Tel +9724854 1415

Fax +97248542477

Email t_saadi@rambam.health.gov.il 


\section{Case report}

A 78-year-old Jewish male patient was hospitalized in our department with a 10-day history of fatigue, nausea, vomiting, diarrhea, anorexia, and abdominal pain. He had also had pruritus and jaundice for 8 days. DM-2 had been recently diagnosed and metformin $850 \mathrm{mg} /$ day had been initiated 2 weeks before presentation to the hospital. The patient reported no alcohol use, smoking, previous liver disease, family history of liver diseases, blood transfusion, exposure to toxins, or cholelithiasis. One month before initiation of metformin treatment, the patient had been given a 13-day amoxicillin-clavulanate (Augmentin) treatment for acute parotitis. His past medical history was significant for hypertension, gout, hyperlipidemia, and diverticulosis. His other medications included aspirin (75 mg/day), pravastatin (20 mg/day), amlodipine ( $5 \mathrm{mg} /$ day), atenolol $(100 \mathrm{mg} /$ day $)$, candesartan $(16 \mathrm{mg} /$ day $)$, and hydrochlorothiazide (12.5 mg/day).

On physical examination, the patient was alert, with prominent jaundice. No stigmata of chronic liver disease were present. There was no pedal edema or ascites. His weight was $82 \mathrm{~kg}$, his height $172 \mathrm{~cm}$, and his body mass index $27.7 \mathrm{~kg} / \mathrm{m}^{2}$. The laboratory findings are summarized in Table 1 . The level of lactic acid was normal. Creatinine, urea, and electrolytes were normal. The levels of glucose during hospitalization were between $120-180 \mathrm{mg} / \mathrm{dL}$. Results of serologic tests were negative for the following: hepatitis B surface antigen; anti-hepatitis B core Immunoglobolin M ( $\operatorname{IgM})$; anti-hepatitis A virus IgM; anti-hepatitis $\mathrm{C}$ virus antibody; anti-Cytomegalovirus IgM; anti-Epstein-Barr virus IgM; anti-nuclear antibodies; antismooth-muscle antibodies; anti-mitochondrial antibodies; and anti-neutrophil cytoplasmic antibodies. Serum ferritin and ceruloplasmin levels were also normal. C-reactive protein level was $9.97 \mathrm{mg} / \mathrm{L}$ (normal range: $0-5 \mathrm{mg} / \mathrm{L}$ ). Complete blood count was normal. An ultrasound and a computerized tomography (CT) scan of the abdomen revealed no abnormalities.

Table I Laboratory test results

\begin{tabular}{lll}
\hline Test & Result & Normal values \\
\hline Total bilirubin $(\mathrm{mg} / \mathrm{dL})$ & 22.2 & $0.2-1$ \\
Direct bilirubin $(\mathrm{mg} / \mathrm{dL})$ & 15.2 & $0-0.4$ \\
Alanine transaminase (U/L) & 1050 & $<40$ \\
Aspartate aminotransferase (U/L) & 496 & $<40$ \\
Alkaline phosphatase (U/L) & $100 \mathrm{I}$ & $30-350$ \\
$\gamma$-glutamyl transpeptidase (U/L) & 1264 & $5-60$ \\
Lactate dehydrogenase (U/L) & 377 & $60-255$ \\
Albumin (g/dL) & 4.2 & $3.5-5$ \\
INR & 0.98 & $0.8-1.2$ \\
\hline
\end{tabular}

Abbreviation: INR, international normalized ratio.
On admission, metformin and pravastatin were discontinued immediately. After 1 week, the patient described significant subjective improvement and the level of bilirubin, alanine transaminase (ALT), aspartate aminotransferase (AST), alkaline phosphatase (ALKP), and $\gamma$-glutamyl transpeptidase (GGT) began to decline gradually. After 9 days of hospitalization, the patient's laboratory results were as follows: total bilirubin, $16.9 \mathrm{mg} / \mathrm{dL}$; direct bilirubin, $12.4 \mathrm{mg} / \mathrm{dL}$; ALT, $308 \mathrm{U} / \mathrm{L}$; AST, $77 \mathrm{U} / \mathrm{L}$; ALKP, $809 \mathrm{U} / \mathrm{L}$; and GGT, $876 \mathrm{U} / \mathrm{L}$. The patient was discharged for outpatient follow-up. Two months following discharge, all hepatobiliary laboratory abnormalities resolved. Thereafter, pravastatin therapy was readministered, and no changes in bilirubin, ALT, AST, ALKP, or GGT levels were observed.

\section{Discussion}

This case report is a useful addition to the short list of literature describing metformin hepatotoxicity available so far. Dealing with a particular patient with diabetes and polypharmacy makes decisions regarding medication termination difficult. A few signs guided our decision to blame metformin for hepatotoxicity in this case. The patient was taking other medications, including pravastatin, for at least 1 year prior to the hepatotoxicity, without adverse sequelae. Furthermore, pravastatin was readministered following resolution of the hepatic abnormalities, without any changes in bilirubin or liver enzyme concentration. The patient's other medications included aspirin ( $75 \mathrm{mg} /$ day), amlodipine ( $5 \mathrm{mg} /$ day), atenolol (100 mg/day), candesartan (16 mg/day), and hydrochlorothiazide $(12.5 \mathrm{mg} / \mathrm{d})$. The patient took these medications for years without adverse sequelae. In addition, liver enzymes and bilirubin returned to normal values after metformin was stopped and despite continuation of the other medications. However, exposure to amoxicillin-clavulanate had occurred 1 month prior to the patient presenting at our hospital. Amoxicillin-clavulanate-associated liver injury has been extensively reported in the literature. ${ }^{12-14} \mathrm{~A}$ recent report from England assessed the incidence of amoxicillin-clavulanateinduced liver injury at 9.91 cases to 100,000 prescriptions. ${ }^{14}$ In another report, from Spain, a mixed cholestatic-hepatocellular pattern of liver injury was associated with older age; the mean time lapse between therapy initiation and jaundice onset was 16 days. ${ }^{13}$ In an attempt to determine the likely cause for acute liver injury in this case - ie, metformin or amoxicillin-clavulanate - we used the Maria and Victorino scale. ${ }^{14}$ This clinical scale is based on (1) the temporal relationship between treatment initiation and the onset of the clinical picture; (2) the exclusion of alternative causes for liver 
Table 2 Case reports published in English with full text on the PubMed database

\begin{tabular}{|c|c|c|c|c|c|c|c|c|}
\hline $\begin{array}{l}\text { Case report } \\
\text { (reference) }\end{array}$ & $\begin{array}{l}\text { Age (years), } \\
\text { gender }\end{array}$ & $\begin{array}{l}\text { Type of } \\
\text { injury }\end{array}$ & $\begin{array}{l}\text { Time to Dx } \\
\text { (weeks) }^{\mathrm{a}}\end{array}$ & Bilirubin ${ }^{b}$ & $\mathrm{ALT}^{\mathrm{b}}$ & AST $^{b}$ & ALKPb & Resolutionc \\
\hline 6 & $67, F$ & Hepatitis & 6 & 4.8 & 905 & $\mathrm{I}, 152$ & 121 & 4 months \\
\hline 10 & $52, F$ & Mixed & 4 & 14.4 & 651 & 583 & 500 & I month \\
\hline 11 & $64, M$ & Cholestasis & 5 & 21.3 & 289 & 214 & 994 & 3 months \\
\hline 17 & $68, M$ & Cholestasis & 4 & NA & NA & NA & NA & NA \\
\hline 18 & $73, \mathrm{~F}$ & Mixed & 3 & 6.5 & 772 & 689 & 635 & 3 weeks \\
\hline 19 & $61, M$ & Hepatitis & 2 & 1.8 & 571 & 623 & 143 & 2 months \\
\hline
\end{tabular}

Notes: ${ }^{\mathrm{a}}$ The time from beginning metformin therapy until the diagnosis was made. ${ }^{\mathrm{b}}$ Maximal values that were reported. The values of bilirubin are in $\mathrm{mg} / \mathrm{dL}$, and those of ALT, AST, and ALKP are in U/L. 'The time from stopping metformin until complete resolution of clinical and laboratory abnormalities.

Abbreviations: ALKP, alkaline phosphatase; ALT, alanine transaminase; AST, aspartate aminotransferase; Dx, diagnosis; F, female; M, male; NA, not available.

injury; (3) extrahepatic manifestations; (4) re-challenging of outcomes; and (5) previous reported cases in the literature. In the present case, amoxicillin-clavulanate scored only 8 points (unlikely), while metformin scored 12 points (possible), thus implicating metformin as the cause for liver injury rather than amoxicillin-clavulanate. Moreover, using the Naranjo adverse drug reaction probability scale - which consists of ten questions that are answered with either "yes," "no," or "do not know," with different point values $(-1,0,+1$, or +2$)$ assigned to each answer - the probability that the symptoms of hepatotoxicity were an adverse drug reaction of metformin was 5 (probable).${ }^{15}$ Therefore, metformin was considered to be the offending medication. Re-challenge was not performed, as it was considered to be inappropriate.

Because metformin is not metabolized in the liver, it has been considered safe from a hepatic standpoint; ${ }^{16}$ however, metformin hepatotoxicity has rarely been reported. Possible mechanisms of injury are direct, idiosyncratic, or a drugdrug interaction ${ }^{11}$ leading to acute hepatocellular and/or cholestatic jaundice.

We searched the PubMed database for English-language publications on metformin hepatotoxicity and found six reports (Table 2): two cases of acute hepatitis, ${ }^{6,19}$ two cases of cholestasis, ${ }^{11,17}$ and two cases of mixed-type (hepatocellular and cholestatic) injury. ${ }^{10,18}$ In all cases, as in our case report, after discontinuation of metformin therapy, the patients' signs and symptoms resolved and the liver enzymes normalized, except for a persistently increased level of alkaline phosphatase in one case, ${ }^{17}$ which was considered likely related to a prolonged cholestatic effect of metformin. No specific treatments were given to these patients. Liver biopsy was performed in three cases, and re-challenge in one case. . $^{6,10,11,17}$

The patient we have described represents the third case report in the literature of mixed hepatocellular and cholestatic liver injury. Discontinuation of metformin treatment led to complete resolution of the patient's signs and symptoms as well as normalization of liver enzymes and bilirubin after 2 months.

\section{Conclusion}

Metformin can induce hepatic injury (hepatocellular and/ or cholestatic), and both physicians and patients should be aware of this potential side effect.

\section{Disclosure}

The authors report no conflicts of interest in this work.

\section{References}

1. Aksay E, Yanturali S, Bayram B, Hocaoğlu N, Kiyan S. A rare side effect of metformin: metformin-induced hepatotoxicity. Turkish Journal of Medical Sciences. 2007;37(3):173-175.

2. Kirpichnikov D, McFarlane SI, Sowers JR. Metformin: an update. Ann Intern Med. 2002;137:25-33.

3. [No authors listed]. Effect of intensive blood-glucose control with metformin on complications in overweight patients with type 2 diabetes (UKPDS 34). UK Prospective Diabetes Study (UKPDS) Group. Lancet. 1998;352:854-865.

4. Glucophage (metformin hydrochloride tablets). Prescribing information. Princeton, NJ: Bristol-Meyers Squibb; 1995.

5. Stumvoll M, Nurjhan N, Perriello G, Dailey G, Gerich JE. Metabolic effects of metformin in non-insulin-dependent diabetes mellitus. $N$ Engl J Med. 1995;333:550-554.

6. Deutsch M, Kountouras D, Dourakis SP. Metformin hepatotoxicity. Ann Intern Med. 2004;140(5):W25-W26.

7. Carrascosa M, Pascual F, Aresti S. Acarbose-induced acute severe hepatotoxicity. Lancet. 1997;349:698-699.

8. Dourakis SP, Tzemanakis E, Sinani C, Kafiri G, Hadziyannis SJ. Gliclazide-induced acute hepatitis. Eur J Gastroenterol Hepatol. 2000;12:119-121.

9. Chitturi S, George J. Hepatotoxicity of commonly used drugs: nonsteroidal anti-inflammatory drugs, antihypertensives, antidiabetic agents, anticonvulsants, lipid-lowering agents, psychotropic drugs. Semin Liver Dis. 2002;22:169-183.

10. Babich MM, Pike I, Shiffman ML. Metformin-induced acute hepatitis. Am J Med. 1998;104:490-492.

11. Desilets DJ, Shorr AF, Moran KA, Holtzmuller KC. Cholestatic jaundice associated with the use of metformin. Am J Gastroenterol. 2001;96:2257-2258.

12. Hussaini SH, O'Brien CS, Despott EJ, Dalton HR. Antibiotic therapy: a major cause of drug-induced jaundice in southwest England. Eur $J$ Gastroenterol Hepatol. 2007;19(1):15-20.

13. Lucena MI, Andrade RJ, Fernández MC, et al; Spanish Group for the Study of Drug-Induced Liver Disease (Grupo de Estudio para las Hepatopatías Asociadas a Medicamentos (GEHAM)). Determinants of the clinical expression of amoxicillin-clavulanate hepatotoxicity: a prospective series from Spain. Hepatology. 2006;44(4): $850-856$. 
14. Maria V, Victorino R. Development and validation of a clinical scale for the diagnosis of drug-induced hepatitis. Hepatology 1997;26: 664-669.

15. Naranjo CA, Busto U, Sellers EM, et al. A method for estimating the probability of adverse drug reactions. Clin Pharmacol Ther. 1981;30: 239-245.

16. Sirtori CR, Franceschini G, Galli-Kienle M, et al. Disposition of metformin (N,N-dimethylbiguanide) in man. Clin Pharmacol Ther. 1978;24:683-693.
17. Nammour F, Fayad N, Peikin S. Metformin-induced cholestatic hepatitis. Endocr Pract. 2003;9(4):307-309.

18. Kutoh E. Possible metformin-induced hepatotoxicity. Am J Geriatr Pharmacother. 2005;3(4):270-273.

19. Cone C, Bachyrycz A, Murata G. Hepatotoxicity associated with metformin therapy in treatment of type 2 diabetes mellitus with nonalcoholic fatty liver disease. Ann Pharmacother. 2010;44(10): $1655-1659$

\section{Publish your work in this journal}

The International Journal of General Medicine is an international, peer-reviewed open-access journal that focuses on general and internal medicine, pathogenesis, epidemiology, diagnosis, monitoring and treatment protocols. The journal is characterized by the rapid reporting of reviews, original research and clinical studies across all disease areas.
A key focus is the elucidation of disease processes and management protocols resulting in improved outcomes for the patient.The manuscript management system is completely online and includes a very quick and fair peer-review system. Visit http://www.dovepress.com/ testimonials.php to read real quotes from published authors. 